\title{
Torsades de pointes in a woman presenting with syncope
}

A 57-year-old woman presented with first onset syncope, which was witnessed by her family members. It occurred in the middle of a conversation and lasted for 30 seconds, followed by spontaneous and prompt recovery. There was upward rolling of eyeballs with no witnessed tongue biting, jerking of limbs or urinary incontinence. There was no family history of sudden cardiac death or heart disease. She denied symptoms of chest pain, palpitation, dyspnoea, diaphoresis and fever. She was not taking any medication or overthe-counter supplements. In addition, there was no identifiable stressors preceding this syncopal episode.

On arrival to hospital, she was afebrile; her blood pressure was $145 / 89 \mathrm{mmHg}$, with a heart rate of $40-70$ beats per minute, respiratory rate of 16 breaths per min and oxygen saturation of $98 \%$ on room air. Initial electrocardiogram (ECG) revealed sinus bradycardia, premature ventricular complexes (PVC), prolonged QT interval (corrected QT [QTc] 600ms), and T inversions in leads aVL and V2-6 (Fig. 1A). Laboratory result revealed normal electrolytes (including potassium, magnesium and calcium), liver function, thyroid function and phaeochromocytoma panel. Her initial highsensitive troponin level was $1,834 \mathrm{ng} / \mathrm{L}$ and peaked at $3,737 \mathrm{ng} / \mathrm{L}$ (normal range $0-18 \mathrm{ng} / \mathrm{L}$ ). Chest radiography showed no consolidation or pleural effusion. Her reverse transcription polymerase chain reaction for COVID-19 came back negative.

Coronary angiogram showed slow flow and mild coronary artery disease. Left ventriculogram revealed apical ballooning with hyperkinesia of the basal anterior and inferior walls (Fig. 1B). Immediate transthoracic echocardiogram (TTE) showed left ventricular ejection fraction (LVEF) of $35 \%$ with wall motion abnormalities (Figs. 1C and D).

During the cardiac catheterisation, patient developed recurrent episodes of torsades de pointes (TdP), and required electrical cardioversion (Fig. 2A). A temporary pacing wire (TPW) was inserted in view of recurrent TdP with a back-up pacing rate set at 80 beats per minute. Cardiac magnetic resonance imaging (CMR) was carried out to exclude reversible causes including myocarditis.

What do Figs. 1 and 2 demonstrate?
A. Arrhythmogenic right ventricular cardiomyopathy
B. Coronary artery dissection
C. Coronary vasospasm
D. Myocarditis
E. Takotsubo cardiomyopathy

CMR showed early oedema in the apex, consistent with diagnosis of takotsubo cardiomyopathy (TCM)

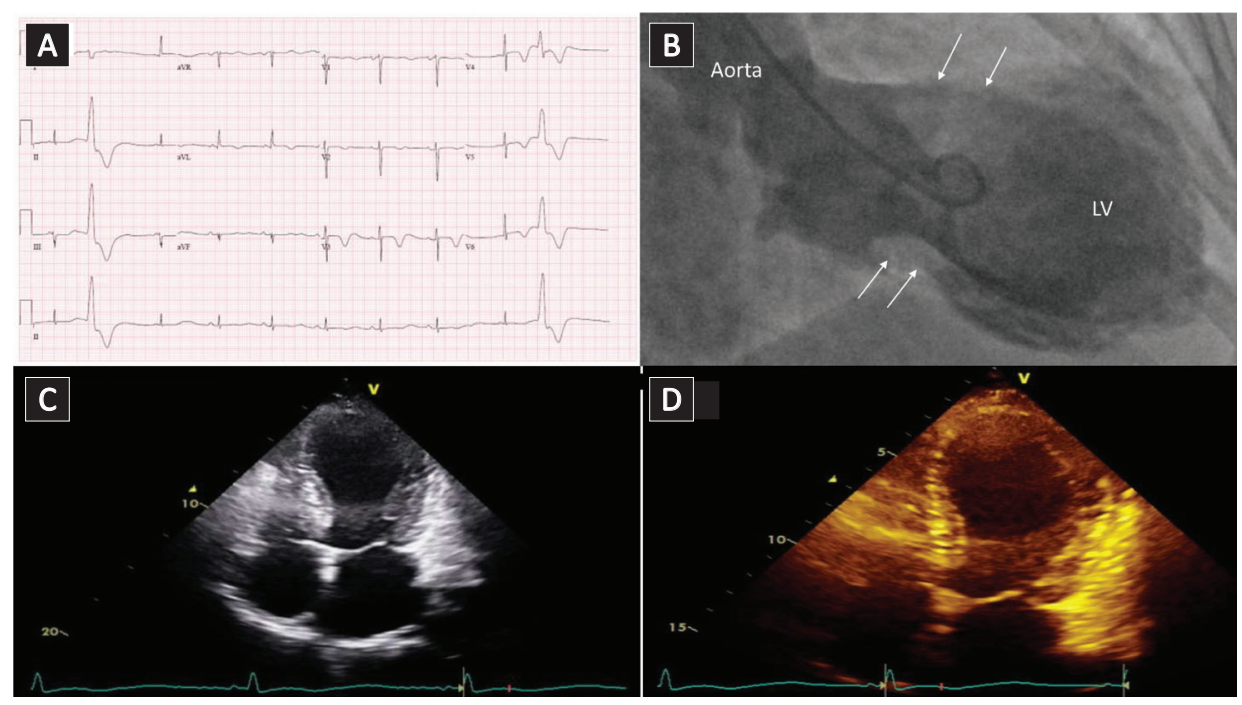

Fig. 1. (A) Initial electrocardiogram showing sinus bradycardia, prolonged QTc $(600 \mathrm{~ms})$ and premature ventricular complexes. (B) Left ventriculogram showing hyperkinesia in the basal segment and akinetic apex (arrows). (C) and (D) Transthoracic echocardiogram showing hypercontractile in the basal segment. QTc: corrected QT 


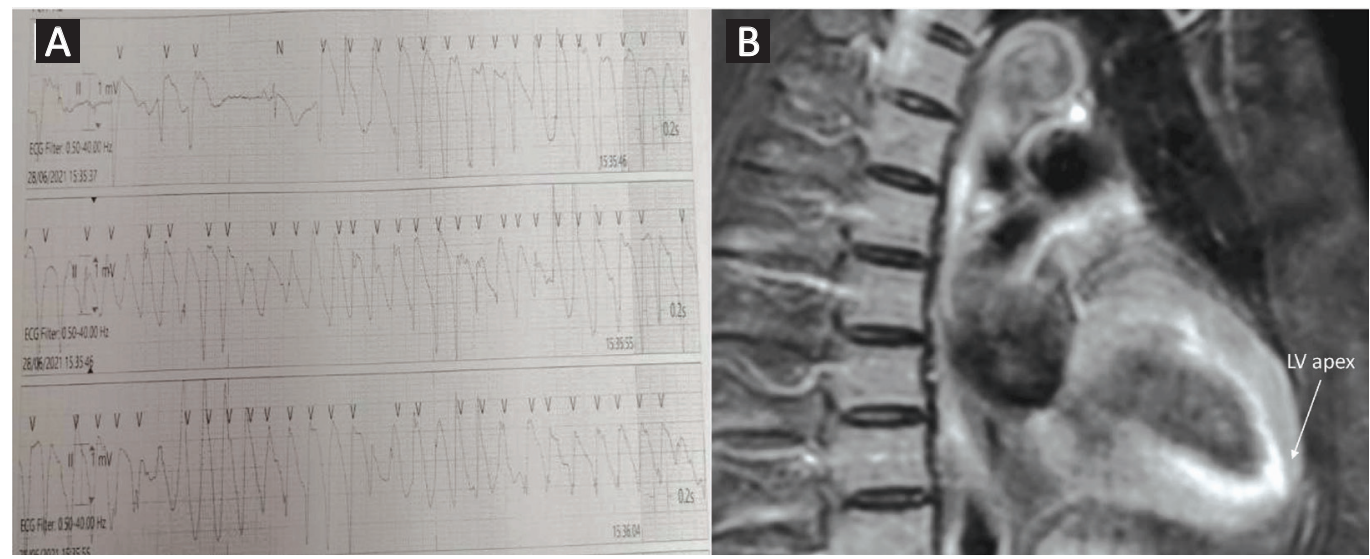

Fig. 2. (A) Telemetry revealing significant torsades de pointes. (B) Cardiac magnetic resonance imaging showing oedema, which is worst in the apical region.

(Fig. 2A). There was no evidence of late gadolinium enhancement or features of myocarditis on CMR.

The patient had regular 12-lead ECGs and her QTc remained lengthened at $500-600 \mathrm{~ms}$ with frequent PVCs seen despite initiation of beta-blockers. Furthermore, when attempting to wean off TPW by reducing the pacing rate to $50 \mathrm{bpm}$, the patient developed TdP. Decision was made for insertion of implantable cardioverter defibrillator (ICD) for secondary prevention. ICD was implanted on day 14 of hospitalisation. She was discharged well on bisoprolol, atorvastatin and aspirin. Subsequent TTE (2 months post-discharge) showed resolution of wall motion abnormalities with recovery of LVEF to 55\%. A follow-up 12-lead ECG showed atrial paced rhythm with QTc of $456 \mathrm{~ms}$ (Fig. 3). Device interrogation did not reveal any episode of ventricular arrhythmia.

Coronary artery dissection and vasospasm are key differential diagnoses to consider in this case. Thus, coronary angiogram remains the gold standard in evaluating coronary anatomy. However, the presentation

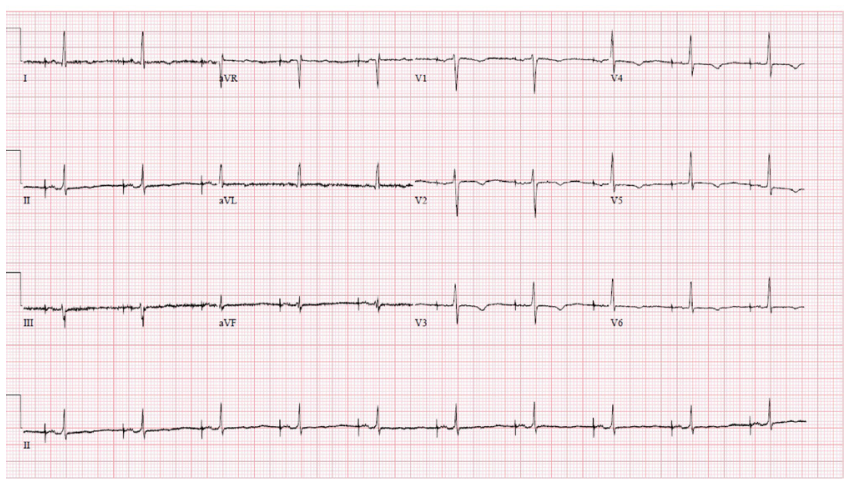

Fig. 3. Electrocardiograph revealing shortening of QTc $(456 \mathrm{~ms})$ and atrial paced rhythm after implantation of implantable cardioverter defibrillator. was atypical as patients would usually complain of chest pain rather than syncope. Myocarditis needs to be excluded in the setting of raised troponin, wall motion abnormalities and patent coronaries. Lake Louise criteria placed a great emphasis on CMR as an imaging modality in diagnosing myocarditis. Arrhythmogenic right ventricular cardiomyopathy is less likely in the absence of right ventricular impairment and dilatation.

This case highlights the association of recurrent episodes of TdP from acquired long QTc syndrome and TCM. Investigation for prolonged QT interval remains challenging as this often involves multiple modalities with a wide range of differential diagnosis to entertain. Female and old age are the only non-modifiable predisposing factors for acquired long QT-syndrome (LQTS). ${ }^{1}$ TCM, despite being a reversible form of cardiomyopathy, may not be benign as this patient presented with syncope with evidence of prolonged QT interval and required an ICD as secondary prevention. It is known that the risk of TdP escalates as QT interval increases. ${ }^{2}$ Early afterdepolarisation-induced triggered activity in the setting of catecholamine excess has been postulated to increase TdP risk. Our patient presented with a substantially prolonged QT interval of $600 \mathrm{~ms}$, which had likely led to cardiogenic syncope from Tdp. The QT interval shortened to $456 \mathrm{~ms}$ after recovery from TCM with no further ventricular arrhythmias detected. Reduction of QT interval $\left(\mathrm{T}_{\text {peak }}-\mathrm{T}_{\text {end }} / \mathrm{QT}\right.$ ratio) dispersion are associated with recovery. ${ }^{3}$

TCM is a rare disorder characterised by transient, reversible left ventricular dysfunction commonly triggered by emotional or physical stress. The US Mayo Clinic criteria are widely adopted by cardiologists in an attempt to better describe the syndrome. ${ }^{4}$ The association between TCM and acquired LQTS have been described in case reports. ${ }^{5}$ However, life- 
threatening arrhythmias (ventricular fibrillation or TdP) in this group of patients are extremely uncommon.

Emotional and physical stressors are recognised triggers for TCM. However, in approximately $30 \%$ of patients, TCM occurred without any evident trigger. ${ }^{6}$ To note, emotional stress or lack of identifiable triggers is more common in women while physical stress leading to TCM is more common in men. There were no identifiable physical or emotional stressors in our index case. Singapore studies have demonstrated that TCM mainly affects post-menopausal women with recent trigger from a surge in catecholamines. ${ }^{7,8}$

Management of TCM remains largely supportive to sustain life and minimise complications until full recovery, which may take weeks. To date, there have been no randomised trials to define optimal treatment of TCM. The use of angiotensin-converting-enzyme inhibitors or angiotensin receptor blockers was associated with improved survival, though the usage of beta-blockers was not. ${ }^{6}$ Temporary transvenous pacing should be considered in the acute setting if patient has TdP. ${ }^{9}$ In this case, there were additional features suggestive of high-risk LQTS (post-TCM QTc $>500 \mathrm{~ms}$, previous syncope and $\mathrm{TdP}$ ), which necessitate the implantation of ICD. ${ }^{10}$

In conclusion, TCM should be recognised as one of the causes of acquired long QT syndrome, in addition to its associated risk of Tdp. There is still much to learn regarding the aetiology and underlying pathophysiology of TCM. Future studies including randomised trials are needed for optimal management of this condition.

\section{REFERENCES}

1. Digby G, Machaalany J, Malik P, et al. Multifactorial QT interval prolongation. Cardiol J 2010;17:184-8.
2. Madias C, Fitzgibbons TP, Alsheikh-Ali A, et al. Acquired long QT syndrome from stress cardiomayopathy is associated with ventricular arrhythmias and torsades de pointes. Heart Rhythm 2011;8:555-61.

3. Mugnai G, Vassanelli F, Pasqualin G, et al. Dynamic changes of repolarization abnormalities in takotsubo cardiomyopathy. Acta Cardiol 2015;70:225-32

4. Prasad A, Lerman A, Rihal CS. Apical ballooning syndrome (Tako-Tsubo or stress cardiomyopathy): a mimic of acute myocardial infarction. Am Heart J 2008;155:408-17.

5. Behr ER, Mahida S. Takotsubo cardiomyopathy and the long-QT syndrome: an insult to repolarization reserve. Europace 2009; 11:697-700.

6. Templin C, Ghadri JR, Diekmann J, et al. Clinical features and outcomes of takotsubo (stress) cardiomyopathy. N Engl J Med 2015;373:929-38

7. Sia $\mathrm{CH}$, Singh D, Loh JP. Can you interpret the ECG under stress? Singapore Med J 2018;59:455-9.

8. Biswas A. Takotsubo cardiomyopathy: an uncommon cause of reversible heart failure. Singapore Med J 2011;52:e59-61.

9. Kurisu S, Inoue I, Kawagoe T, et al. Torsade de pointes associated with bradycardia and takotsubo cardiomyopathy. Can J Cardiol 2008;24:640-2.

10. Denney SD, Lakkireddy DR, Khan IA. Long QT syndrome and torsade de pointes in transient left ventricular apical ballooning syndrome. Int J Cardiol 2005;100:499-501.

Shiun Woei Wong ${ }^{1,2,3}{ }_{M R C P}(U K)$, Shonda $\operatorname{Ng}^{1}{ }_{M R C P}(U K)$, Joo Hor Tan ${ }^{1}$ MRCP (UK)

${ }^{1}$ Department of Cardiology, Tan Tock Seng Hospital, Singapore

${ }^{2}$ Lee Kong Chian School of Medicine, Nanyang Technological University, Singapore

${ }^{3}$ Yong Loo Lin School of Medicine, National University of Singapore, Singapore

Correspondence: Dr Shiun Woei Wong, Department of Cardiology, Tan Tock Seng Hospital, 11 Jalan Tan Tock Seng, Singapore 308433. Email: shiunwoei@gmail.com 